\title{
DC and AC Josephson effects with superfluid Fermi atoms across a Feshbach resonance
}

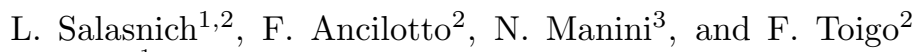 \\ ${ }^{1}$ CNR-INFM and CNISM, Unità di Padova, \\ Via Marzolo 8, 35122 Padova, Italy \\ ${ }^{2}$ Dipartimento di Fisica "Galileo Galilei" and CNISM, \\ Università di Padova, \\ Via Marzolo 8, 35122 Padova, Italy \\ ${ }^{3}$ Dipartimento di Fisica, \\ ETSF and CNISM, Università di Milano, \\ Via Celoria 16, 20133 Milano, Italy
}

\begin{abstract}
We show that both DC and AC Josephson effects with superfluid Fermi atoms in the BCS-BEC crossover can be described at zero temperature by a nonlinear Schrödinger equation (NLSE). By comparing our NLSE with mean-field extended BCS calculations, we find that the NLSE is reliable in the BEC side of the crossover up to the unitarity limit. The NLSE can be used for weaklylinked atomic superfluids also in the BCS side of the crossover by taking the tunneling energy as a phenomenological parameter.
\end{abstract}

\section{INTRODUCTION}

Few years ago the AC Josephson effect [1, 2] with atomic superfluids was predicted [3] and observed [4] with Bose-Einstein Condensates (BECs). AC Josephson oscillations in superfluid atomic Fermi gases have been considered theoretically by several authors [5, 6, 67, 8]. Recently, Spuntarelli, Pieri and Strinati [9] have studied the DC Josephson effect [1, 2] across the BCS-BEC crossover with neutral fermions by using the extended BCS equations: they computed the current-phase relation throughout the BCS-BEC crossover at zero temperature for a two-spin component Fermi gas in the presence of a barrier [9].

In this paper we discuss a nonlinear Schrödinger equation (NLSE) [8, 10, 11] which is equivalent to the equations of superfluid hydrodynamics [12] with the inclusion of a gradient term of the von Weizsäcker type [13, 14, 15]. By using the NLSE we reproduce the DC Josephson results of Spuntarelli, Pieri and Strinati [9], in the BEC side of the BCS-BEC crossover, i.e. from the unitarity region to the deep BEC regime. However, we show that our NLSE describes [8] both DC and AC Josephson effects [1, 2] also in the BCS regime of two weakly-linked atomic superfluids if the tunneling energy is taken as a phenomenological parameter à la Feynmann [16, 17].

\section{HYDRODYNAMICS OF FERMI SUPERFLUIDS AT ZERO-TEMPERATURE}

At zero temperature the hydrodynamics equations of a two-component fermionic superfluid made of atoms of mass $m$ are given by

$$
\begin{gathered}
\frac{\partial}{\partial t} n+\nabla \cdot(n \mathbf{v})=0 \\
m \frac{\partial}{\partial t} \mathbf{v}+\nabla\left[\frac{1}{2} m v^{2}+U(\mathbf{r})+\mu\left(n, a_{F}\right)\right]=0
\end{gathered}
$$

where $n(\mathbf{r}, t)$ is the local density and $\mathbf{v}(\mathbf{r}, t)$ is the local superfluid velocity. Here $n(\mathbf{r}, t)=n_{\uparrow}(\mathbf{r}, t)+n_{\downarrow}(\mathbf{r}, t)$, with $n_{\uparrow}(\mathbf{r}, t)=n_{\downarrow}(\mathbf{r}, t)$ and $\mathbf{v}(\mathbf{r}, t)=\mathbf{v}_{\uparrow}(\mathbf{r}, t)=\mathbf{v}_{\downarrow}(\mathbf{r}, t) . U(\mathbf{r})$ is the external potential and $\mu\left(n, a_{F}\right)$ is the bulk chemical potential, i.e. the zero-temperature equation of state of the uniform system, which depends on the fermion-fermion scattering length $a_{F}$. The density $n(\mathbf{r}, t)$ is such that

$$
N=\int n(\mathbf{r}, t) d^{3} \mathbf{r}
$$

is the total number of atoms in the fluid. In fact, due to the absence of the normal component, the superfluid density coincides with the total density and the superfluid current with the total current.

Equations (11) and (2) are nothing but the Euler equations of an inviscid and irrotational fluid. Since $\mathbf{v}$ is irrotational, it can be written as the gradient of a scalar field. The connection between superfluid hydrodynamics and quantum 
mechanics is made by the formula

$$
\mathbf{v}=\frac{\hbar}{2 m} \nabla \theta
$$

where $\theta(\mathbf{r}, t)$ is the phase of the condensate wave-function

$$
\Xi(\mathbf{r}, t)=|\Xi(\mathbf{r}, t)| e^{i \theta(\mathbf{r}, t)}=\left\langle\hat{\psi}_{\uparrow}(\mathbf{r}, t) \hat{\psi}_{\downarrow}(\mathbf{r}, t)\right\rangle,
$$

with $\hat{\psi}_{\sigma}(\mathbf{r}, t)$ the fermionic field operator with spin component $\sigma=\uparrow, \downarrow[18,19]$. Notice the factor $2 m$ (Cooper pairs) instead of $m$ in Eq. (4) [12, 18, 19].

The equations of superfluid hydrodynamics describe quite accurately static properties and low-energy collective modes of oscillation of wavelength $\lambda \gg \xi$, where $\xi$ is the healing length of the superfluid. Recently, Combescot, Kagan and Stringari [20] have suggested that

$$
\xi=\frac{\hbar}{m v_{c r}}
$$

where $v_{c r}$ is the critical velocity of the Landau criterion for dissipation [18, 20]. According to Combescot, Kagan and Stringari [20], in the BEC regime of bosonic dimers $v_{c r}$ coincides with the sound velocity, i.e.

$$
v_{c r}=c_{s}=\sqrt{\frac{n}{m} \frac{\partial \mu}{\partial n}}
$$

Instead, in the BCS regime $v_{c r}$ is related to the breaking of Cooper pairs through the formula

$$
v_{c r}=\sqrt{\frac{\sqrt{\mu^{2}+|\Delta|^{2}}-\mu}{m}},
$$

where $|\Delta|$ is the energy gap of Cooper pairs [20] Note that the equations of superfluid hydrodynamics (10) and (22) do not take into account the effect of pair breaking.

In the BCS-BEC crossover the bulk chemical potential of Eq. (2) can be written as

$$
\mu\left(n, a_{F}\right)=\frac{\hbar^{2}}{2 m}\left(3 \pi^{2} n\right)^{2 / 3}\left(f(y)-\frac{y}{5} f^{\prime}(y)\right)
$$

where $f(y)$ is a dimensionless universal function of the inverse interaction parameter

$$
y=\frac{1}{k_{F} a_{F}}
$$

where $k_{F}=\left(3 \pi^{2} n\right)^{1 / 3}$ is the Fermi wavenumber [10]. One can parametrize $f(y)$ as follows:

$$
f(y)=\alpha_{1}-\alpha_{2} \arctan \left(\alpha_{3} y \frac{\beta_{1}+|y|}{\beta_{2}+|y|}\right),
$$

where the values of the parameters $\alpha_{1}, \alpha_{2}, \alpha_{3}, \beta_{1}, \beta_{2}$, reported in Ref. [10], are fitting parameters based on asymptotics and fixed-node Monte-Carlo data [21]. We will call in the following Monte-Carlo equation of state (MC EOS) the equation $\mu=\mu\left(n, a_{F}\right)$ obtained from (9) and (11).

Within the mean-field extended BCS theory [19, 22], the bulk chemical potential $\mu$ and the gap energy $\Delta$ of the uniform Fermi gas are instead found by solving the following extended BCS (EBCS) equations [22, 23]

$$
\begin{aligned}
& -\frac{1}{a_{F}}=\frac{2(2 m)^{1 / 2}}{\pi \hbar^{3}} \Delta^{1 / 2} \int_{0}^{\infty} d y y^{2}\left(\frac{1}{y^{2}}-\frac{1}{\sqrt{\left(y^{2}-\frac{\mu}{\Delta}\right)^{2}+1}}\right) \\
& n=\frac{N}{V}=\frac{(2 m)^{3 / 2}}{2 \pi^{2} \hbar^{3}} \Delta^{3 / 2} \int_{0}^{\infty} d y y^{2}\left(1-\frac{\left(y^{2}-\frac{\mu}{\Delta}\right)}{\sqrt{\left(y^{2}-\frac{\mu}{\Delta}\right)^{2}+1}}\right) .
\end{aligned}
$$

By solving these two EBCS equations one obtains the chemical potential $\mu$ as a function of $n$ and $a_{F}$ in the full BCS-BEC crossover (see for instance Ref. [23]). Note that EBCS theory does not predict the correct BEC limit: the molecules have scattering length $a_{M}=2 a_{F}$ instead of $a_{M}=0.6 a_{F}$ [12]. We call EBCS equation of state (EBCS EOS) the mean-field equation of state $\mu=\mu\left(n, a_{F}\right)$ obtained from Eqs. (12) and (13). Of course, the MC EOS is much closer than the EBCS EOS to the MC results obtained in Ref. [21]. 


\section{SUPERFLUID NLSE FOR THE BCS-BEC CROSSOVER}

Inspired by the Ginzburg-Landau theory [24], by the density functional theory (DFT) [25], and by the low-energy effective field theory (EFT) [26, 27], we introduce the complex wave function

$$
\Psi(\mathbf{r}, t)=\sqrt{\frac{n(\mathbf{r}, t)}{2}} e^{i \theta(\mathbf{r}, t)}
$$

which describes boson-like Cooper pairs with the normalization

$$
\int|\Psi(\mathbf{r}, t)|^{2} d^{3} \mathbf{r}=\frac{N}{2}
$$

that is different from the normalization of the condensate wave function $\Xi(\mathbf{r}, t)[23]$ while the phase $\theta(\mathbf{r}, t)$ is the same [18, 19]. We now look for the simplest nonlinear Schrödinger equation of $\Psi(\mathbf{r}, t)$ which satisfies Eq. (4) and reproduces the equations of superfluid hydrodynamics in the classical limit $(\hbar \rightarrow 0)$. We find [8, 11] that the nonlinear Schrödinger equation

$$
i \hbar \frac{\partial}{\partial t} \Psi(\mathbf{r}, t)=\left[-\frac{\hbar^{2}}{4 m} \nabla^{2}+2 U(\mathbf{r})+2 \mu\left(n(\mathbf{r}, t), a_{F}\right)\right] \Psi(\mathbf{r}, t)
$$

gives the equations of superfluid hydrodynamics in the classical limit $(\hbar \rightarrow 0)$. For finite $\hbar$, this superfluid NLSE adds to the classical hydrodynamics equations a quantum pressure term

$$
T_{Q P}=-\frac{\hbar^{2}}{8 m} \frac{\nabla^{2} \sqrt{n}}{\sqrt{n}}
$$

containing explicitly the Planck constant $\hbar$ (gradient correction in DFT, next-to-leading correction in low-energy EFT) [11]. Note that in the deep BEC regime from Eq. (16) one recovers the familiar Gross-Pitaevskii equation for Bose-condensed dimers (molecules of two fermions), where

$$
\mu\left(n, a_{F}\right)=\frac{4 \pi \hbar^{2} a_{d d}\left(a_{F}\right)}{2 m} n
$$

with $a_{d d}\left(a_{F}\right)$ the dimer-dimer scattering length, which depends on the fermion-fermion scattering length $a_{F}$. Within the mean-field theory one finds $a_{d d}\left(a_{F}\right)=2 a_{F}$, whereas four-body theory and also MC many-body data yield $a_{d d}\left(a_{F}\right)=0.6 a_{F}[12]$.

\section{DIRECT CURRENT JOSEPHSON EFFECT}

We use our time-dependent superfluid NLSE (16) to study the direct-curent (DC) Josephson effect [1, 2]. Consider a square-well barrier

$$
U(\mathbf{r})= \begin{cases}V_{0} & \text { for }|z|<d \\ 0 & \text { elsewhere }\end{cases}
$$

which separates the superfluid into two regions, and assume a stationary solution

$$
\Psi(\mathbf{r}, t)=\Phi(\mathbf{r}) e^{i \theta(\mathbf{r})} e^{-i 2 \bar{\mu} t / \hbar}
$$

with constant and uniform number supercurrent

$$
J=n(\mathbf{r}) \mathbf{v}(\mathbf{r})=2 \Phi(\mathbf{r})^{2} \frac{\hbar}{2 m} \nabla \theta(\mathbf{r}) .
$$

From the previous equation it follows $(\nabla \theta)^{2}=m^{2} J^{2} /\left(\hbar^{2} \Phi^{4}\right)$ and also

$$
\left[-\frac{\hbar^{2}}{4 m} \nabla^{2}+\frac{m}{4} \frac{J^{2}}{\Phi(\mathbf{r})^{4}}+2 U(\mathbf{r})+2 \mu\left(n(\mathbf{r}), a_{F}\right)\right] \Phi(\mathbf{r})=2 \bar{\mu} \Phi(\mathbf{r}) .
$$




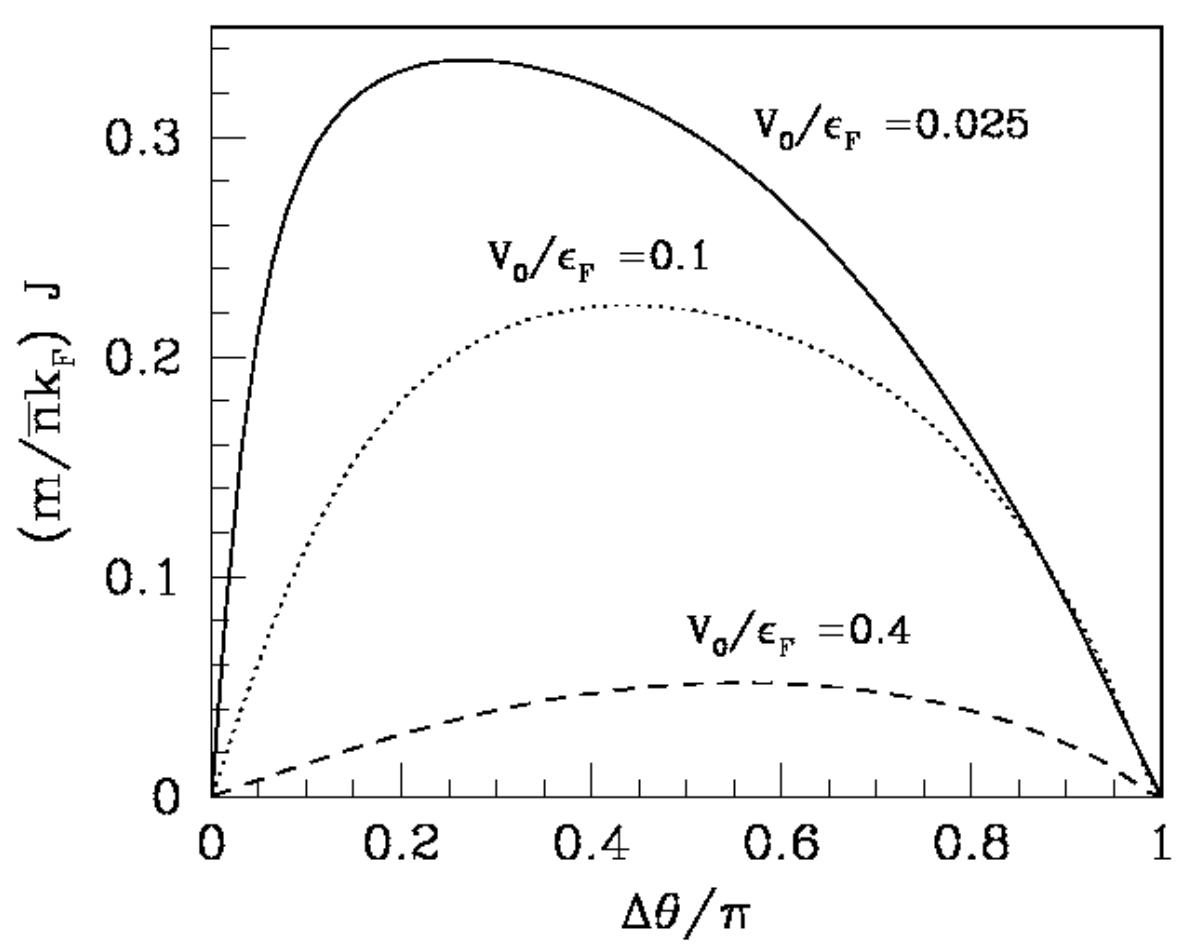

FIG. 1: DC Josephson current $J$ vs phase difference $\Delta \theta$ for a superfluid Fermi gas at unitarity $(y=0)$, for three values of the energy barrier $V_{0} . \epsilon_{F}=\hbar^{2}\left(3 \pi^{2} \bar{n}\right)^{2 / 3} /(2 m)$ is the Fermi energy. The width of the barrier is $L=4 / k_{F}$, where $k_{F}=\left(3 \pi^{2} n\right)^{1 / 3}$ is the Fermi wave number.

We solve this stationary superfluid NLSE by imposing a constant and uniform density $\bar{n}$ at infinity:

$$
\Phi(\mathbf{r}) \rightarrow \sqrt{\frac{\bar{n}}{2}} \text { for }|\mathbf{r}| \rightarrow \infty
$$

Given $\Phi(\mathbf{r})$ at fixed $J$, the phase $\theta(\mathbf{r})$ is then obtained from

$$
\theta(\mathbf{r})=\theta\left(\mathbf{r}_{0}\right)+\frac{m J}{\hbar} \int_{\mathbf{r}_{0}}^{\mathbf{r}} \frac{1}{\Phi(\mathbf{r})^{2}} d \mathbf{r}
$$

The phase difference across the barrier is defined as

$$
\Delta \theta=\theta(z=+\infty)-\theta(z=-\infty) .
$$

This conditions allows us to establish the relationship between the current $J$ and the phase difference $\Delta \theta$. Figure 1 reports our results obtained by using the bulk EBCS EOS at unitarity $\left(a_{F}= \pm \infty\right)$. As expected we recover the Josephson equation

$$
J=J_{0} \sin (\Delta \theta)
$$

in the regime of high barrier (small tunneling, weak-link). In the limit of very small barrier (quasi-free transport, strong-link) $J_{0}$ has its maximum value, given by

$$
J_{0}^{\max }=\bar{n} v_{c r}
$$

where $v_{c r}$ is the Landau critical velocity introduced in the previous section [20].

In Fig. 2 we plot $J_{0}^{\max }$ as a function of the inverse interaction parameter $y=1 /\left(k_{F} a_{F}\right)$, and compare our data (curves) with the results of Spuntarelli, Pieri and Strinati (symbols) [9]. Note that the data of Spuntarelli, Pieri and Strinati [9] have been obtained by solving the full self-consistent Bogoliubov-de Gennes equations of the EBCS theory 


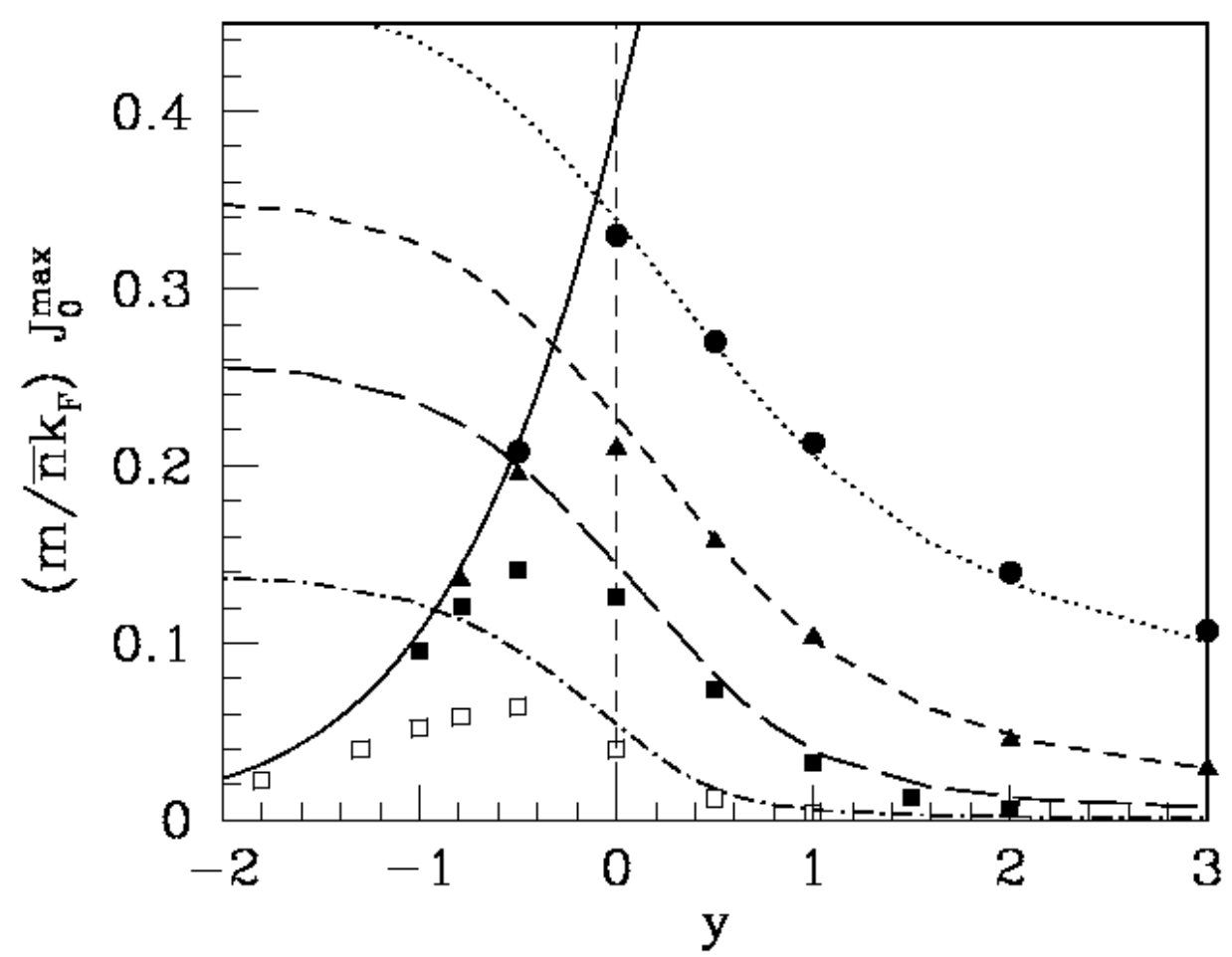

FIG. 2: Maximum Josephson current $J_{0}^{\max }$ vs inverse interaction parameter $y=1 /\left(k_{F} a_{F}\right)$ in the BCS-BEC crossover. Solid curve: $J_{0}^{\max }$ based on pair breaking in the BCS regime [9, 20]. Other curves: superfluid NLSE. Symbols: microscopic mean-field calculations of Ref. [9]. Four values of the energy barrier $V_{0} / \epsilon_{F}$ are considered: $0.025,0.10,0.2,0.4$. The width of the barrier is $L=4 / k_{F}$.

in the presence of the barrier (19). Figure 2 shows that the NLSE reproduces the DC Josephson results of Ref. [9], but only in the right side of the BCS-BEC crossover, i.e. from the unitarity limit to the deep BEC regime. It is not surprising that the NLSE fails in the BCS regime $(y<0)$, since it neglects completely the effect of pair breaking. The critical velocity $v_{c r}$ predicted by NLSE is the sound velocity $c_{s}$ also in the BCS regime. Nevertheless, it is remarkable that the NLSE works quite well in the BEC regime $(y>0)$ up to the unitarity limit $(y=0)$.

\section{ALTERNATE CURRENT JOSEPHSON EFFECT}

We now consider a high barrier (small tunneling, weak-link), without imposing a constant supercurrent $J$ and discuss the alternate-current (AC) Josephson effect [1, 2] with superfluid Fermi atoms [8]. We start from the time-dependent superfluid NLSE (16) and look for a time-dependent solution of the form

$$
\Psi(\mathbf{r}, t)=c_{A}(t) \Phi_{A}(\mathbf{r})+c_{B}(t) \Phi_{B}(\mathbf{r}),
$$

where $\Phi_{A}(\mathbf{r})$ and $\Phi_{B}(\mathbf{r})$ is the quasi-stationary solutions normalized to one and localized in region $A$ and $B$ respectively. In this way we obtain [8] the following two-state model

$$
\begin{aligned}
i \hbar \frac{\partial}{\partial t} c_{A}(t) & =E_{A}(t) c_{A}(t)+K c_{B}(t) \\
i \hbar \frac{\partial}{\partial t} c_{B}(t) & =E_{B}(t) c_{B}(t)+K c_{A}(t)
\end{aligned}
$$

for the two complex coefficients $c_{A}(t)$ and $c_{B}(t)$, related to the number of atoms in the two regions. In our two-state model, $E_{A}(t)$ is the time-dependent energy in region $A$, given by

$$
E_{A}(t) \simeq \int \Phi_{A}(\mathbf{r})\left[-\frac{\hbar^{2}}{4 m} \nabla^{2}+2 U(\mathbf{r})+2 \mu\left(2\left|c_{A}(t)\right|^{2} \Phi_{A}(\mathbf{r})^{2}, a_{F}\right)\right] \Phi_{A}(\mathbf{r}) d^{3} \mathbf{r} .
$$




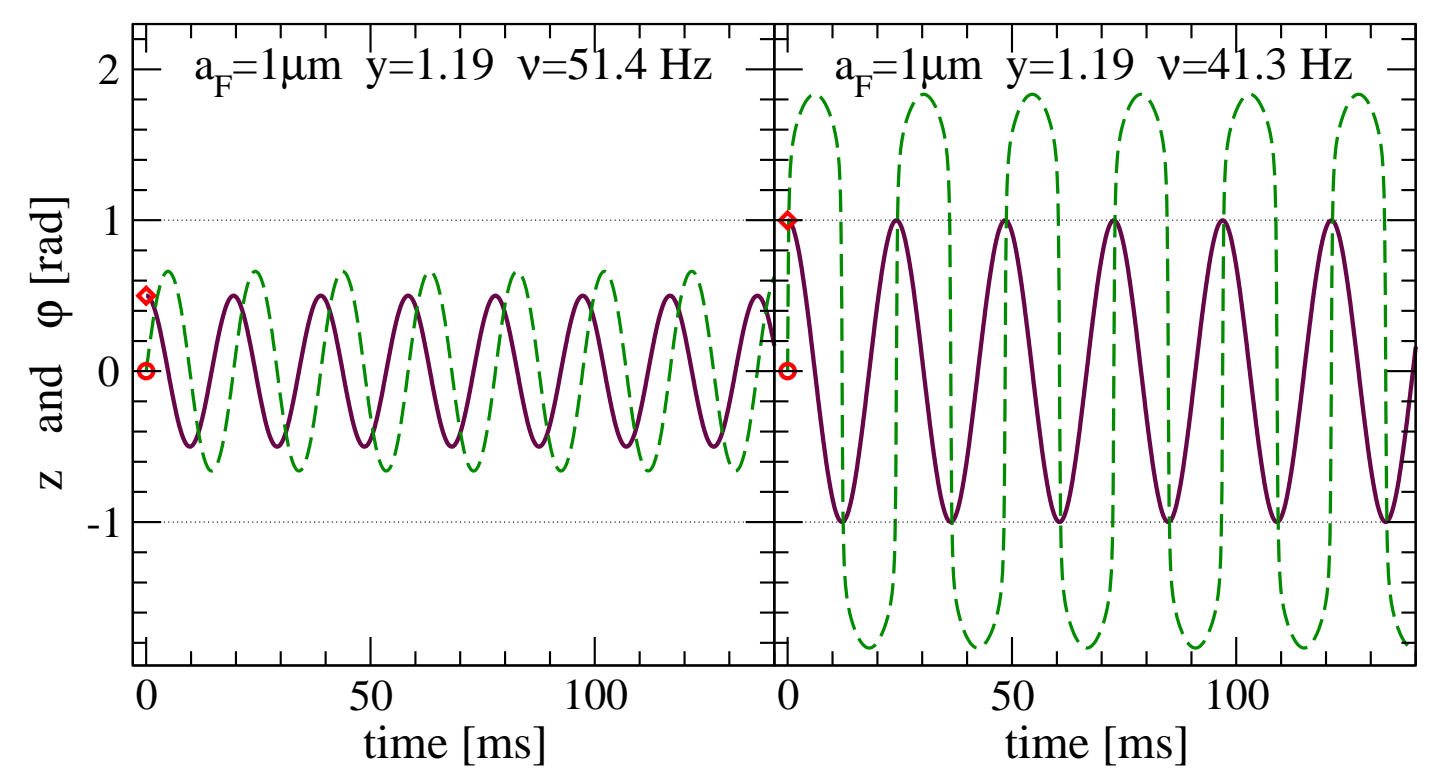

FIG. 3: Zero-mode in the AC Josephson effect by solving AJJ equations. $N=10^{6}{ }^{40} \mathrm{~K}$ atoms between two symmetric regions of volume $25 \cdot 10^{6} \mu \mathrm{m}^{3}$, tunneling parameter $K / k_{\mathrm{B}}=10^{-9}$ Kelvin and Fermi-Fermi scattering length $a_{F}=1 \mu \mathrm{m}$, corresponding to $y=1.19$. Solid line: population imbalance $z(t)$; dashed line: phase difference $\varphi(t)$. Initial conditions: $\varphi(0)=0$ and $z(0)=0.5$ (left); $\varphi(0)=0$ and $z(0)=0.999$ (right). Adapted from Ref. [8].

There is obviously a similar expression for the time-dependent energy $E_{B}(t)$. The constant coupling energy $K$ describes instead the tunneling between the two regions $\mathrm{A}$ and $\mathrm{B}$ :

$$
K \simeq \int \Phi_{A}(\mathbf{r})\left[-\frac{\hbar^{2}}{4 m} \nabla^{2}+2 U(\mathbf{r})\right] \Phi_{B}(\mathbf{r}) d^{3} \mathbf{r} .
$$

From our previous analysis of the DC Josephson effect, we expect that this expression is correct only in the right side of the BCS-BEC crossover. However, to extend the study the Josephson effect to the left side of the BCS-BEC crossover one may use $K$ in Eqs. (29) and (30) as a phenomenological parameter à la Feynman [16, 17].

We can write the complex coefficient $c_{A}(t)$ as

$$
c_{A}(t)=\sqrt{\frac{N_{A}(t)}{2}} e^{i \theta_{A}(t)}
$$

with $N_{A}(t)$ number of atoms and $\theta(t)$ phase in region $A$. Again, a similar expression holds for $c_{B}(t)$. In terms of the phase difference

$$
\varphi(t)=\theta_{B}(t)-\theta_{A}(t)
$$

and relative number imbalance

$$
z(t)=\frac{N_{B}(t)-N_{A}(t)}{N_{A}(t)+N_{B}(t)}=\frac{N_{B}(t)-N_{A}(t)}{N}
$$

the two-mode equations give

$$
\begin{aligned}
& \dot{z}(t)=-\frac{2 K}{\hbar} \sqrt{1-z(t)^{2}} \sin \varphi(t) \\
& \dot{\varphi}(t)=\frac{2}{\hbar}\left[\mu\left(\frac{N}{2 V}(1+z(t))\right)-\mu\left(\frac{N}{2 V}(1-z(t))\right)\right]+\frac{2 K}{\hbar} \frac{z(t)}{\sqrt{1-z(t)^{2}}} \cos \varphi(t) .
\end{aligned}
$$

These are the atomic Josephson junction (AJJ) equations describing the oscillations of $N$ Fermi atoms tunneling in the superfluid state between region $A$ and region $B$, of equal volume $V$ [8]. Notice that equations (36) and (37) 


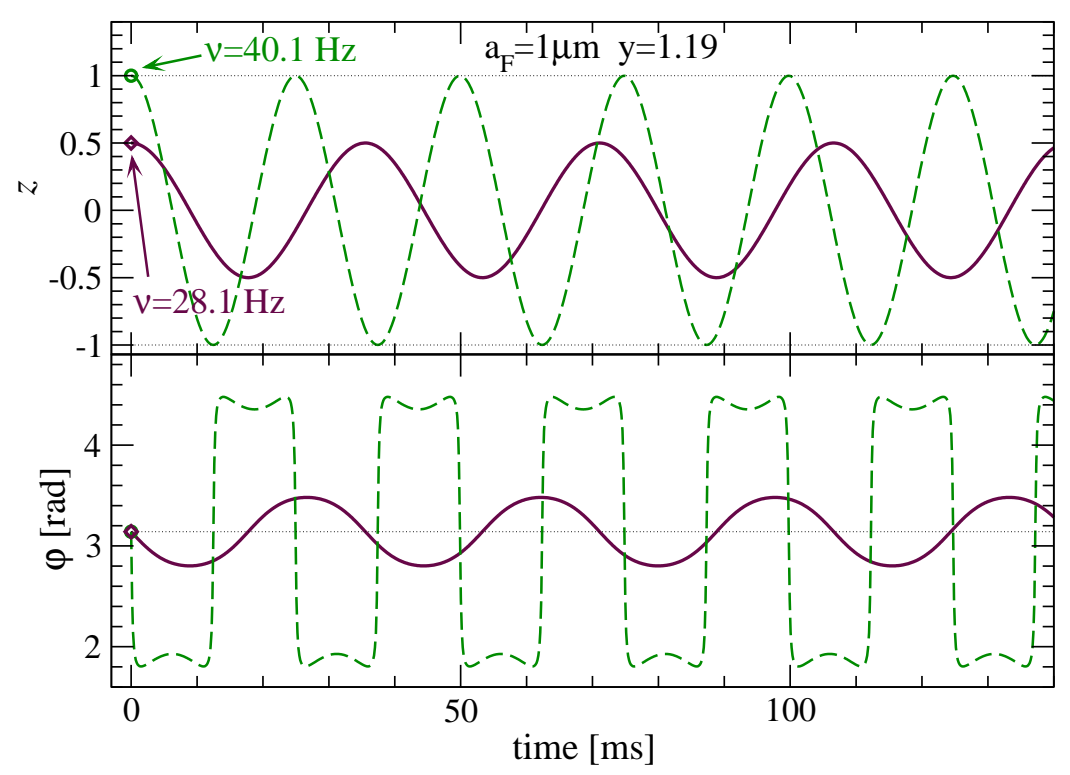

FIG. 4: $\pi$-mode in the AC Josephson effect by solving AJJ equations. All physical parameters are the same as in Fig. 3. Upper panel: population imbalance $z(t)$; lower panel: phase difference $\varphi(t)$. Initial conditions: $\varphi(0)=0$ and $z(0)=0.5($ solid); $\varphi(0)=0$ and $z(0)=0.999$ (dashed).

generalize the BJJ equations obtained by Smerzi et al. [3] for Bose-Einstein condensates. Moreover, these equations, linking the tunneling current

$$
J=-\dot{z} \frac{N}{2}=\frac{K N}{\hbar} \sqrt{1-z^{2}} \sin \varphi=J_{0} \sqrt{1-z^{2}} \sin \varphi
$$

to the phase difference $\varphi$, reduce to the familiar Josephson expression $J=J_{0} \sin (\varphi)$ in the appropriate limit $|z| \ll 1$.

The nonlinear AJJ equations can be linearized around the stable stationary solution

$$
\bar{z}=0 \quad \text { and } \quad \bar{\varphi}=2 \pi j
$$

where $j$ is an integer. In this way one finds the following frequency of small oscillation

$$
\nu_{0}=\frac{K}{\pi \hbar} \sqrt{1+\frac{2 m c_{s}^{2}}{K}}
$$

which is called zero-mode. Here $c_{s}$ is the sound velocity computed at the mean density $n=N / V$ of the superfluid. This zero-mode is the analog of the Josephson plasma oscillation in superconducting junctions [2]. Figure 3 reports the zero-mode oscillations of $z(t)$ and $\varphi(t)$, in the case of ${ }^{40} \mathrm{~K}$ atoms setting $a_{F}=1 \mu \mathrm{m}$. The oscillation starting from $z(0)=0.5$ indicates that the solution (40) of the linearized equations (36) and (37) are fairly accurate even for finite and not quite small amplitude. Eventually however, for very large amplitude, $z(0)=0.999$, deviations from the harmonic approximation become quite visible.

We observe that the AJJ equations (36) and (37) produce also a $\pi$-mode solution with $\bar{z}=0$ and $\bar{\varphi}=\pi(2 j+1)$ and the self-trapping solution with population imbalance $(\bar{z} \neq 0)$ (for details see $[8]$ ). Figure 4 reports $\pi$-mode oscillations for two different initial unbalance. Note the significant frequency increase and waveshape distortion induced by nonlinear effects.

\section{CONCLUSIONS}

This paper reviews a superfluid NLSE providing the hydrodynamic equations of Fermi superfluids plus a gradient correction. Both hydrodynamics equations and superfluid NLSE are reliable to investigate static properties and lowenergy collective dynamics. It is important to stress that the equations of superfluid hydrodynamics are nothing else than the time-dependent local density approximation (LDA) for an irrotational system. The advantage of using the NLSE is that can take into account surface and shape effects beyond LDA, and these can be relevant for a small 
number of particles [28]. We have shown that in the study of the DC Josephson effect, the superfluid NLSE works quite well at the right side (BEC regime) of the BCS-BEC crossover up to the unitarity limit of infinite scattering length. In addition, we have suggested that for studying DC and AC Josephson effects in weakly-linked atomic superfluids the NLSE can be used in the full BCS-BEC crossover within the two-mode approximation. In this case the tunneling energy coefficient must be taken as a phenomenological parameter at the left side (BCS regime) of the crossover.

We thank Andrea Spuntarelli and Pierbiagio Pieri for making available their data [9]. LS acknowledges a reseach grant from GNFM-INdAM. This work has been partially supported by Fondazione CARIPARO.

[1] B.D. Josephson, Phys. Lett. 1, 251 (1962).

[2] A. Barone and G. Paternò, Physics and Applications of the Josephson Effect (Wiley, New York, 1982).

[3] A. Smerzi, S. Fantoni, S. Giovanazzi, and S.R. Shenoy, Phys. Rev. Lett. 79, 4950 (1997).

[4] M. Albiez, R. Gati, J. Fölling, S. Hunsmann, M. Cristiani, and M.K. Oberthaler, Phys. Rev. Lett. 95, 010402 (2005); F.S. Cataliotti, S. Burger, C. Fort, P. Maddaloni, F. Minardi, A. Trombettoni, A. Smerzi, and M. Inguscio, Science 293, 843 (2001).

[5] Gh.-S. Paraoanu, M. Rodriguez, and P. Tormä, Phys. Rev. A 66, 041603(R) (2002).

[6] M. Wouters, J. Tempere, and J.T. Devreese, Phys. Rev. A 70, 013616 (2004).

[7] S.K. Adhikari, Eur. Phys. J. D 47, 413 (2008).

[8] L. Salasnich, N. Manini, and F. Toigo, Phys. Rev. A 77, 043609 (2008).

[9] A. Spuntarelli, P. Pieri, and G.C. Strinati, Phys. Rev. Lett. 99, 040401 (2007).

[10] N. Manini and L. Salasnich, Phys. Rev. A 71, 033625 (2005); G. Diana, N. Manini, and L. Salasnich, Phys. Rev. A 73, 065601 (2006); L. Salasnich and N. Manini, Laser Phys. 17, 169 (2007).

[11] L. Salasnich, e-preprint arXiv:0804.1277.

[12] S. Giorgini, L.P. Pitaevskii, and S. Stringari, arXiv:0706.3360.

[13] C.F. von Weizsäcker, Z. Phys. 96, 431 (1935).

[14] D.A. Kirzhnits, Sov. Phys. JEPT 5, 64 (1957); D.A. Kirzhnits, Field Theoretical Methods in Many-Body Systems (Pergamon Press, London, 1967).

[15] L. Salasnich, J. Phys. A: Math. Theor. 40, 9987 (2007).

[16] R.P. Feynman, R. Leighton, and M. Sands, Feynman Lectures On Physics, vol. 3 (Addison Wesley, Reading, 1966); R.P. Feynman, Statistical Mechanics. A set of Lectures (Benjamin, Reading, 1973).

[17] R.E. Packard, Rev. Mod. Phys. 70, 641 (1998).

[18] L.D. Landau and E.M. Lifshitz, Statistical Physics: Theory of the Condensed State, (Pergamon, London, 1987).

[19] A.J. Leggett, Quantum Liquids (Oxford Univ. Press, Oxford, 2006).

[20] R. Combescot, M. Yu. Kagan, and S. Stringari, Phys. Rev A 74, 042717 (2006).

[21] G.E. Astrakharchik et al., Phys. Rev. Lett. 93, 200404 (2004).

[22] M. Marini, F. Pistolesi, and G.C. Strinati, Eur. Phys. J. B 1, 151 (1998).

[23] L. Salasnich, N. Manini, and A. Parola, Phys. Rev. A 72, 023621 (2005); L. Salasnich, Phys. Rev. A 76, 015601 (2007).

[24] V.L. Ginzburg and L.D. Landau, Zh. Eksp. Teor. Fiz. 20, 1064 (1950).

[25] R.M. Dreizler and E.K.U. Gross, Density functional theory (Springer-Verlag, Berlin, 1990).

[26] M.P. Kemoklidze and L.P. Pitaevskii, Sov. Phys. JEPT 23, 160 (1966); S. De Palo, C. Castellani, C. Di Castro, and B. K. Chakraverty, Phys. Rev. B 60, 564 (1999); P. Pieri, and G.C. Strinati, Phys. Rev. Lett. 91030401 (2003).

[27] D.T. Son and M. Wingate, Ann. Phys. 321, 197 (2006); G. Rupak and T. Schäfer, e-preprint arXiv:0804.2678v2.

[28] S.K. Adhikari and L. Salasnich, "Density functional theory for cigar-shaped and disk-shaped Fermi superfluids at unitarity", submitted for publication (2008). 